\title{
Estimation of the Reliability of Automatic Axial-balancing Devices for Multistage Centrifugal Pumps
}

\author{
Ivan Pavlenko1, Justyna Trojanowska², Oleksandr Gusak³, Vitalii Ivanov*, Jan Pitel ${ }^{5}$, Vita Pavlenko ${ }^{6}$ \\ ${ }^{1}$ Department of General Mechanics and Machine Dynamics, Faculty of Technical Systems and Energy Efficient Technologies, \\ Sumy State University, 2 Rymskogo-Korsakova St., Sumy, 40007, Ukraine \\ 2 Department of Management and Production Engineering, Faculty of Mechanical Engineering and Management, Poznan \\ University of Technology, 5 M. Sklodowska-Curie Sq., Poznan, 60965, Poland \\ ${ }^{3}$ Department of Applied Hydro- and Aeromechanics, Faculty of Technical Systems and Energy Efficient Technologies, Sumy State \\ University, 2 Rymskogo-Korsakova St., Sumy, 40007, Ukraine \\ ${ }^{4}$ Department of Manufacturing Engineering, Machines and Tools, Faculty of Technical Systems and Energy Efficient Technologies, \\ Sumy State University, 2 Rymskogo-Korsakova St., Sumy, 40007, Ukraine \\ ${ }^{5}$ Department of Industrial Engineering and Informatics, Faculty of Manufacturing Technologies with a seat in Presov, Technical \\ University of Kosice, 1 Bayerova St., Presov, 08001, Slovak Republic \\ ${ }^{6}$ Machine-building College of Sumy State University, 17 Shevchenka Ave., Sumy, 40000, Ukraine \\ *Corresponding author, e-mail: ivanov@tmvi.sumdu.edu.ua
}

Received: 04 July 2018, Accepted: 29 October 2018, Published online: 13 December 2018

\begin{abstract}
The permanent increase of operating parameters of modern multistage centrifugal pumps requires more precise modelling for estimation their dynamic reliability and needs to develop new approach and methodology for the related calculations. It is necessary to change the approach assuming empirical coefficients to another one based on the probabilistic design for analysis of reliability for pumps and their nodes. In this case, the probabilistic parameters of the design features and fluid flow are significant for the calculation (e. g. geometry of gap seals, boundary conditions, material properties) and should be considered. The automatic axial-balancing device of the rotor is one of the most important nodes of multi-stage centrifugal pumps. It operates as the balancing disc for the axial force up to $1 \mathrm{MN}$. This paper presents the methodology for the numerical calculation of the balancing disc and its influence on the reliability of multistage centrifugal pumps. The proposed approach is based on the criterion of the permissible amplitudes of axial oscillations of the rotor with the automatic axial-balancing device considering probabilistic origin of main geometrical, hydromechanical and operating parameters.
\end{abstract} Keywords

erosion wear, axial oscillations, amplitude-frequency response, probabilistic calculation, mathematical expectation, probability density function

\section{Introduction}

For balancing of total axial forces in multistage centrifugal pumps, the automatic axial-balancing devices are used. Designing these assemblies requires the possibility of carrying out precise calculations including investigations of their efficiency that affects reliability of the pump. Increasing the reliability of a pumping system is primarily related to the requirements of safety standards following an increase in the operating parameters of these machines. Prevention of unreliability of pumping installations requires raising the standards for the strength, criteria for assessing the dynamic state of machines and their efficiency.
The gradual deterioration of operating parameters of the balancing disc is caused by gradual erosion of the walls of seals and bearing supports. When the width of the transverse gap is too small, dry friction may occur, which reduces the mechanical efficiency of the pump. When the gap is too large, the hydraulic losses increase. Moreover, accidentally changed geometrical and hydromechanical parameters change the dynamic state of the machine. The amplitude of axial oscillations may exceed the range specified in traditional calculations that leads to the occurrence of dry friction in the axial gap, increases mechanical losses and accelerates wear of rings of the balancing disc. 
Due to the abovementioned, the automatic axial-balancing device requires the possibly of precise calculation that treat the reliability problem, and can be the subject of new original design solutions.

\section{Literature Review}

The influence of hydraulic resistance on bearing life of multistage centrifugal pumps is analyzed in work [1]. The methodology of experimental research is presented in paper [2]. The need of improvement of the pump and compressor equipment's reliability is proved in paper [3].

Contribution of the back-vane radius to the axial thrust is investigated in works $[4,5]$ from the experimental data, and the variation of thrust values due to changing back vane height and clearance is obtained. Improving modular fixtures for ensuring locating accuracy in shaft machining is proposed in paper [6].

Application of the numerical simulation for static and dynamic analysis of the automatic axial-balancing device of the rotor for multistage centrifugal pumps, and new design of balancing disc are presented in papers [7]. General approach for dynamic analysis of the rotor supported on ball bearings using two- and three-dimensional finite element models is presented in work [8]. Brand-new design of automatic balancing device is proposed in the certificate of authorship [9]. Application of artificial neural network for identification of bearing stiffness characteristics in rotor dynamics analysis is proposed in the paper [10]. Effective modes of axial balancing of centrifugal pump rotor are obtained, as well as new design solutions of balancing devices based on hydrostatic seals, as well as the development of the procedure for numerical simulation of the automatic balancing device under the condition of minimal energy losses are presented in paper [11]. General approach for using artificial neural networks for dynamic analysis of the rotors is presented in work [12].

Methodology for upgrading rotors of pumps and compressors using combined technology of electroerosive alloying is presented in the paper [13].

However, the approaches proposed in abovementioned papers do not allow taking into account probabilistic origin of geometrical, hydromechanical and operating parameters (radial gap, presence of closing environment, composition of solid microparticles in a liquid, material properties of wearing surfaces, reversibility of axial thrust, etc.).

The following research is aimed at the new approach that allows to estimate reliability of automatic axial-balancing devices for multistage centrifugal pumps, as well

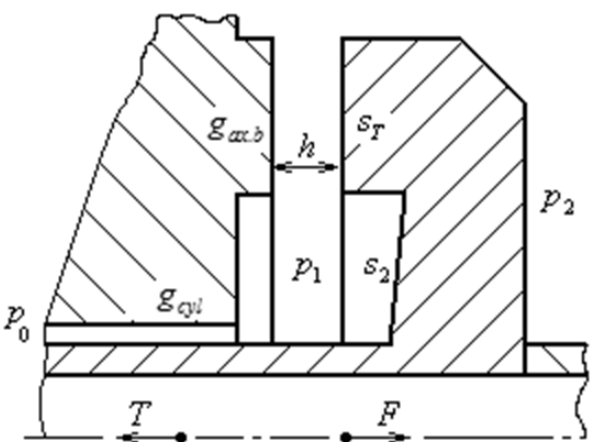

Fig. 1 Design scheme of the automatic balancing device

as to obtain results in a range of the confidence interval for acceptable amplitudes of rotor oscillations.

\section{Research Methodology}

Reliable operating of the balancing disc can be considered, when axial vibrations, leakages of the fluid (hydraulic losses) and friction are not exceeded the critical level. Dynamic analysis of the automatic axial-balancing device is based on the solution of the equation of rotor axial movement, fluid flow continuity equations through the radial and axial gaps considering the compressibility of the liquid and its displacement due to changes of the axial gap (Fig. 1):

$\left\{\begin{array}{l}m \ddot{z}+\mu \dot{z}+c z=F-T ; \\ g_{c y l} \sqrt{p_{0}-p_{1}}=g_{a x . b} u^{3 / 2} \sqrt{p_{1}-p_{2}}+\frac{V_{2}}{E} \dot{p}_{1}+s_{2} \dot{z},\end{array}\right.$

where $m$ - mass of the rotor assembly; $\mu, c, E$ - damping factor, stiffness factor, modulus of elasticity of the liquid layer in the radial gap; $z, u$-axial displacement of the rotor and its dimensionless value; $T$ - total axial forces acting on the rotor of the multistage centrifugal pump; $F$ - balancing force acting on the balancing disc; $g_{c y l}, g_{a x . b}$ - conductivities of the radial and axial gaps; $p_{0}$ - pressure after the last stage of the pump; $p_{1}, p_{2}$ - pressures in the chambers in front of the balancing disc and behind it; $s_{2}$ - area between the hub and inner diameter of the radial gap; $s_{e}$ - equivalent surface of the balancing disc; $V_{2}$ - volume of the chamber in front of the shield.

The initial system of the differential equations (Eq. (1)) is nonlinear with respect to parameters $z$ and $p_{1}$. The application of the calculus of variations and introducing dimensionless parameters allow obtaining the following system of linear differential equations:

$\left\{\begin{array}{l}\delta \ddot{u}+2 n \delta \dot{u}+\omega_{0}^{2} \delta u-a_{1} \delta \psi_{1}=-a_{2} \delta \tau ; \\ a_{4} \delta \dot{u}+b_{2} \delta u+a_{3} \delta \dot{\psi}_{1}+b_{1} \delta \psi_{1}=0\end{array}\right.$ 
where $\delta \tau, \delta u, \delta \psi$ - dimensionless variations of the axial force, axial gap and pressure in the chamber in front of the balancing disc; $a_{1}, \ldots, a_{4}, b_{1}, b_{2}, n, \omega_{0}$ - coefficients and parameters of the automatic axial-balancing device defined by the following dependencies:

$$
\begin{aligned}
a_{1} & =\frac{s_{e} p_{b}}{m z_{b}} ; \quad a_{2}=\frac{p_{b} s_{b}}{m z_{b}} ; \quad a_{3}=\frac{V_{2}}{E g_{c y l}} ; \quad a_{4}=\frac{s_{2} z_{b}}{g_{c y l} p_{b}} ; \\
b_{1} & =\frac{g_{a x . b}}{g_{c y l}} \frac{u^{3 / 2}}{2 \sqrt{\psi_{10}}}+\frac{1}{2 \sqrt{\psi_{0}-\psi_{10}}} ; \quad b_{2}=\frac{3}{2} \frac{g_{a x . b}}{g_{c y l}} \cdot \sqrt{u_{0} \psi_{0}} ; \\
n & =\frac{\mu}{2 m} ; \quad \omega_{0}=\sqrt{\frac{c}{m}}
\end{aligned}
$$

where $z_{b}, p_{b}, s_{b}-$ nominal (basic) width of the axial gap, pressure in front of the balancing disc and after it; $u_{0}, \psi_{0}$, $\psi_{10}$ - dimensionless axial gap and pressure determined under the conditions of axial equilibrium in the case of stationary operating of the automatic axial-balancing device.

The system of differential equations (Eq. (2)) can be rewritten in the matrix form:

$\left[\begin{array}{cc}p^{2}+2 n p+\omega_{0}^{2} & -a_{1} \\ a_{4} p+b_{2} & a_{3} p+b_{1}\end{array}\right]\left\{\begin{array}{c}\delta u \\ \delta \psi\end{array}\right\}=\left\{\begin{array}{c}-\delta \tau \\ 0\end{array}\right\}$,

where $p=d / d t$ - differentiation operator with respect to time.

The transfer function for the hydromechanical "system rotator - balancing disc" is determined by solving the determinant of the matrix Eq. (4):

$W(p)=\frac{\delta u}{\delta \tau}=\frac{\left(a_{3} p+b_{1}\right) / a_{2}}{\left(p^{2}+2 n p_{1}+\omega_{0}^{2}\right)\left(a_{3} p+b_{1}\right)+a_{1}\left(a_{4} p+b_{2}\right)}$.

The substitution $p=i \cdot \omega$ to the transfer function $(i-$ imaginary unit) allows to obtain frequency transfer function $W(i \omega)$. Its module and phase angle are amplitude and phase frequency responses $A(\omega), \varphi(\omega)$ respectively (Fig. 2).

Reliable operating state of the automatic axial-balancing device corresponds to the condition that dimensionless axial gap $u$ is within its minimum and maximum limits $\left[u_{\min }, u_{\max }\right]$ in the area $u-A$. The effective work of the balancing disc is carried out under the condition that the point $M$ in the area $D_{1}$ is bounded by lines corresponding to the given changes of the axial gap in a range $\left[h_{c y l}^{\min }, h_{c y l}^{\max }\right]$ (Fig. 3).

Diversity of durability of components of this design as a result of the application of many calculation techniques requires a transition from the analysis including factors determining the permissible loads of its individual elements to calculations based on the assumed probability of

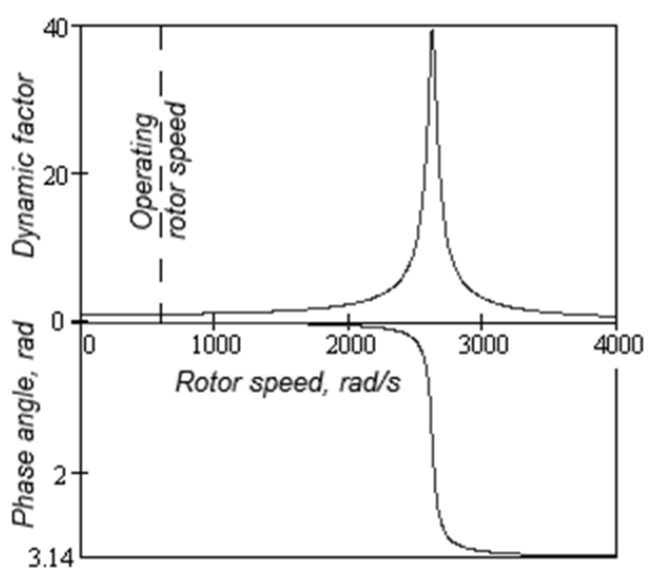

Fig. 2 Amplitude and phase frequency responses of the automatic axial-balancing device

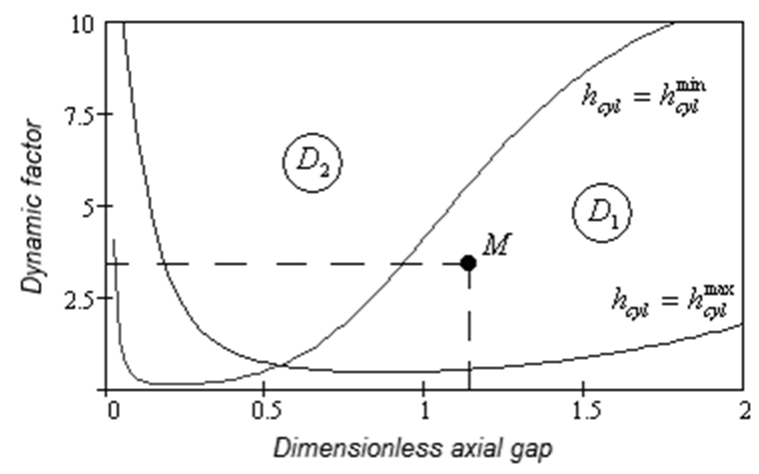

Fig. 3 Operating areas of the automatic axial-balancing device of the rotor: $D_{1}$ - optimal operating area; $D_{2}$ - unacceptable operating area

their reliable operation, i. e. a new approach for designing. As a result, the efficient work of the automatic axial-balancing device is characterized by the average inter-renovation lifetime.

Changes of the relative width of the radial gap in time can be described by the following dependence:

$\chi(t)=\chi_{0}+\dot{\chi} t=a+b t$,

where $a, b$ - statistically independent coefficients, which are independent of time.

Because the erosion rate is affected by a large number of parameters (mechanical properties of the material of radial and axial surfaces, machining accuracy of the components, roughness, waviness, presence of solid microparticles $n$ the pumped liquid, etc.), the probability of changes of coefficients $a$ and $b$ can be assumed in the form of Gaussian normal distribution according to the main central theorem [14]:

$p_{a}(a)=\frac{1}{\sqrt{2 \pi} \sigma_{a}} e^{-\frac{\left(a-a_{0}\right)^{2}}{2 \sigma_{a}^{2}}} ; \quad p_{b}(b)=\frac{1}{\sqrt{2 \pi} \sigma_{b}} e^{-\frac{\left(b-b_{0}\right)^{2}}{2 \sigma_{b}^{2}}}$, 
where $a_{0}, b_{0}$ - mathematical expectations determined by approximation of the curve of the erosion of the radial gap or obtained empirically by measuring the geometry of the radial gap in time; $\sigma_{a}=k \cdot a_{0}, \sigma_{b}=k \cdot b_{0}$ - standard deviations of $a$ and $b$ calculated using the least squares method on the basis of available statistical data using the variation coefficient $k$.

The probability distribution of the function $\chi(t)$ can be determined using the probability distribution of a set of two statistically independent quantities $a$ and $b$ :

$p_{\chi}(\chi, t)=p_{\chi}(a+b t)=\frac{1}{\sqrt{2 \pi} \sigma_{\chi}(t)} e^{-\frac{[\chi-\bar{\chi}(t)]^{2}}{2 \sigma_{\chi}^{2}(t)}}$,

where the mathematical expectation and standard deviation of $\chi(t)$ are:

$\bar{\chi}(t)=a_{0}+b_{0} t ; \quad \sigma_{\chi}(t)=\sqrt{\sigma_{a}^{2}+\sigma_{b}^{2} t^{2}}$.

The cumulative probability density function of the radial gap, as well as the probability density function of the total axial force by the assumption of the Gaussian normal distribution can be written in the following forms:

$F_{\chi}(\chi, t)=\int_{-\infty}^{\chi} p_{\chi}(\chi, t) d \chi ; \quad p_{\varphi}(\varphi)=\frac{1}{\sqrt{2 \pi} \sigma_{\varphi}} e^{-\frac{\left[\varphi-\varphi_{0}\right]^{2}}{2 \sigma_{\varphi}^{2}}}$,

where $\varphi_{0}$ and $\sigma_{\varphi}=k \cdot \varphi_{0}$ - mathematical expectation and standard deviation coupled with the variation coefficient $k$.

As a result, the cumulative probability density function

$F_{\varphi}(\varphi)=\int_{-\infty}^{\varphi} p_{\varphi}(\varphi) d \varphi$.

Probability of occurrence of the point $M$ in area $B$ of reliable operating state of the pump can be specified by the following formula:

$P_{B}(t)=\int_{\varphi_{\min }}^{\varphi_{\max }}\left[F_{\chi}\left(\chi_{2}, t\right)-F_{\chi}\left(\chi_{1}, t\right)\right] \frac{\partial F_{\varphi}(\varphi)}{\partial \varphi} d \varphi$.

Average lifetime of the automatic axial-balancing device is calculated by the following formula:

$T=\int_{0}^{\infty} P_{B}(t) d t$.

\section{Results}

The following calculations are available for the next initial data for the multistage centrifugal pump. Radial gap radius $r_{0}=40 \mathrm{~mm}$; inner and outer radiuses of axial gap:
Table 1 Overall calculation results, hr

\begin{tabular}{lccc}
\hline $\begin{array}{l}\text { Material (according } \\
\text { to the US Standard) }\end{array}$ & $\begin{array}{c}\text { ASTM A48 } \\
\text { Class 30 }\end{array}$ & $\begin{array}{c}\text { UNS } \\
\text { S42000 }\end{array}$ & $\begin{array}{c}\text { UNS } \\
\text { G41300 }\end{array}$ \\
\hline $\begin{array}{l}\text { Average lifetime } \\
\text { (maintenance interval) }\end{array}$ & 7300 & 36400 & 54600 \\
$\begin{array}{l}\text { Time for reliable operation } \\
\text { with the quantile } 0.8\end{array}$ & 5500 & 27400 & 41100 \\
\hline
\end{tabular}

$r_{1}=80 \mathrm{~mm}, r_{2}=120 \mathrm{~mm}$; radial gap $h_{0}=100 \mu \mathrm{m}$; radial gap length $l_{\text {cyl }}=40 \mathrm{~mm}$; minimal and maximum axial gaps: $h_{\min }=50 \mu \mathrm{m}, h_{\max }=200 \mu \mathrm{m}$; pressure $p_{0}=10 \mathrm{MPa}$; rotor speed $3000 \mathrm{rpm}$.

The amplitude and phase frequency responses are presented on Fig. 2. The dependence between the amplitude of axial oscillations of the rotor with the balancing disc and random change of the axial gap width due to erosion is presented on Fig. 3. Overall calculation results as average lifetime and time of reliable operation are presented in Table 1. Maximum value of the average lifetime corresponds to the use of stainless steel UNS G41300 as the material of rings of the balancing disc, and the minimum one is related to the use of cast iron ASTM A48 Class 30.

\section{Conclusions}

The presented approach allows determining the average operating time of the balancing disc using the statistical data of the wear for axial gap. The results of numerical calculation of the efficiency for the automatic axial-balancing device are obtained considering the proposed criterion of optimal operating time.

The influence of the material of the balancing disc on the reliable operating time is analyzed. Particularly, for the specified parameters of a pump, the reliable operating time is in a range of 5 500-54 $600 \mathrm{hr}$ depending on the following materials of axial surfaces: cast iron (ASTM A48 Class 30) and stainless steels (UNS S42000, UNS G41300). It is stated that dynamic factor for axial oscillations of the rotor for operating speed $3000 \mathrm{rpm}$ does not exceed 1.2 with the quantile 0.8 .

Finally, the approach for estimating reliability of automatic axial-balancing devices for multistage centrifugal pumps according to the acceptable level of rotor oscillations has been approved.

\section{Acknowledgement}

The achieved results are obtained as a result of the joint collaboration between Sumy State University (Ukraine), Poznan University of Technology (Poland) and Technical 
University of Kosice (Slovak Republic), as well as within the recent research of the Department of General Mechanics and Machine Dynamics of Sumy State University "Investigation

\section{References}

[1] Godbole, V., Patil, R., Gavade, S. S. "Axial Thrust in Centrifugal Pumps - Experimental Analysis", In: Proceedings of the $15^{\text {th }}$ International Conference on Experimental Mechanics, ICEM15, Porto, Portugal, 2977, 2012.

[2] Szwedowicz, J., Senn, S. M., Abhari, R. S. "Optimum Strain Gage Application to Bladed Assemblies", Journal of Turbomachinery, 124(4), pp. 606-613, 2002.

https://doi.org/10.1115/1.1506957

[3] Liaposhchenko, O. O., Sklabinskyi, V. I., Zavialov, V. L., Pavlenko, I. V., Nastenko, O. V., Demianenko, M. M. "Appliance of Inertial Gas-Dynamic Separation of Gas-Dispersion Flows in the Curvilinear Convergent-Divergent Channels for Compressor Equipment Reliability Improvement", IOP Conference Series: Materials Science and Engineering, 233, 012025, 2017. https://doi.org/10.1088/1757-899X/233/1/012025

[4] Schaefer, G., Olson, E. "Experimental evaluation of axial thrust in pumps", Journal of World Pumps, 393, pp. 34-37, 1999.

[5] Gantar, M., Florjancic, D., Sirok, B. "Hydraulic Axial Thrust in Multistage Pumps - Origins and Solutions", Journal of Fluids Engineering, 124(2), pp. 336-341, 2001. https://doi.org/10.1115/1.1454110

[6] Karpus, V. E., Ivanov, V. A. "Locating accuracy of shafts in V-blocks", Russian Engineering Research, 32(2), pp. 144-150, 2012. https://doi.org/10.3103/S1068798X1202013X

[7] Pavlenko, I. "Static and Dynamic Analysis of the Closing Rotor Balancing Device of the Multistage Centrifugal Pump", Applied Mechanics and Materials, 630, pp. 248-254, 2014. https://doi.org/10.4028/www.scientific.net/AMM.630.248

[8] Pavlenko, I. V., Simonovskiy, V. I., Demianenko, M. M. "Dynamic analysis of centrifugal machines rotors supported on ball bearings by combined application of 3D and beam finite element models", IOP Conference Series: Materials Science and Engineering, 233, $012053,2017$.

https://doi.org/10.1088/1757-899X/233/1/012053

[9] Martsynkovskyy, V.A., Deineka, A., Korczak, A., Peczkis, G. "Static Calculation of the Rotor Unloading Automatic Machine for a High-Pressure Centrifugal Pump", IOP Conference Series: Materials Science and Engineering, 233, 012056, 2017. https://doi.org/10.1088/1757-899X/233/1/012056 of rotor dynamics for turbopump units and reciprocating compressor plants" (State Reg. No. 0117U004922).

[10] Pavlenko, I., Simonovskiy, V., Ivanov, V., Zajac, J., Pitel, J. "Application of Artificial Neural Network for Identification of Bearing Stiffness Characteristics in Rotor Dynamics Analysis", In: Ivanov, V., Rong, Y., Trojanowska, J., Venus, J., Liaposhchenko, O., Zajac, J., Pavlenko, I., Edl, M., Perakovic, D. (eds) Advances in Design, Simulation and Manufacturing, DSMIE 2018, Lecture Notes in Mechanical Engineering, Springer, Cham, Switzerland, pp. 325-335, 2019. https://doi.org/10.1007/978-3-319-93587-4_34

[11] Kalinichenko, P., Gusak, O., Khovanskyy, S., Krutas, Y. "Substantiation and development of the procedure for calculating a hydraulic balancing device under condition of minimal energy losses", Eastern-European Journal of Enterprise Technologies, 2(7), pp. 36-41, 2017. https://doi.org/10.15587/1729-4061.2017.97162

[12] Pavlenko, I., Trojanowska, J., Ivanov, V., Liaposhchenko, O. "Scientific and Methodological Approach for the Identification of Mathematical Models of Mechanical Systems by Using Artificial Neural Networks", In: Machado, J., Soares, F., Veiga, G. (eds) Innovation, Engineering and Entrepreneurship, HELIX 2018, Lecture Notes in Electrical Engineering, 505, Springer, Cham, Switzerland, pp. 299-306, 2019. https://doi.org/10.1007/978-3-319-91334-6_41

[13] Tarelnyk, V., Martsynkovskyy, V. "Upgrading of Pump and Compressor Rotor Shafts Using Combined Technology of Electroerosive Alloying", Applied Mechanics and Materials, 630, pp. 397-412, 2014 https://oi.org/10.4028/www.scientific.net/AMM.630.397

[14] Svetlitsky, V. A. "Statistical Dynamics and Reliability: Theory for Mechanical Structures", 1st ed., Springer-Verlag, Berlin, Heidelberg, Germany, 2003. https://doi.org/10.1007/978-3-540-45826-5 